\title{
3D structure of the dihydropyridine receptor of skeletal muscle
}

\author{
Montserrat Samsó \\ Department of Physiology and Biophysics, Virginia Commonwealth University, Richmond, VA, \\ USA
}

\begin{abstract}
Excitation contraction coupling, the rapid and massive $\mathrm{Ca}^{2+}$ release under control of an action potential that triggers muscle contraction, takes places at specialized regions of the cell called triad junctions. There, a highly ordered supramolecular complex between the dihydropyridine receptor (DHPR) and the ryanodine receptor (RyR1) mediates the quasi-instantaneous conversion from T-tubule depolarization into $\mathrm{Ca}^{2+}$ release from the sarcoplasmic reticulum (SR). The DHPR has several key modules required for EC coupling: the voltage sensors and II-III loop in the alpha1s subunit, and the beta subunit. To gain insight into their molecular organization, this review examines the most updated 3D structure of the DHPR as obtained by transmission electron microscopy and image reconstruction. Although structure determination of a heteromeric membrane protein such as the DHPR is challenging, novel technical advances in protein expression and 3D labeling facilitated this task. The 3D structure of the DHPR complex consists of a main body with five irregular corners around its perimeter encompassing the transmembrane alpha $1 \mathrm{~s}$ subunit besides the intracellular beta subunit, an extended extracellular alpha 2 subunit, and a bulky intracellular II-III loop. The structural definition attained at $19 \AA$ resolution enabled docking of the atomic coordinates of structural homologs of the alpha1s and beta subunits. These structural features, together with their relative location with respect to the RyR1, are discussed in the context of the functional data.
\end{abstract}

Key Words: dihydropyridine receptor, excitation-contraction coupling, transmission electron microscopy, 3D reconstruction

Eur J Tranls Myol - Basic Appl Myol 2015; 25 (1): 27-33

The process by which an action potential propagating through the muscle plasma membrane results into $\mathrm{Ca}^{2+}$ release from the sarcoplasmic reticulum (SR) and subsequent muscle contraction is called excitation-contraction (EC) coupling. In skeletal muscle of vertebrates this process takes place in the triad junction, where the transverse-tubule (TT) extensions of the plasma membrane, orthogonal to the main axis of the muscle fiber, run adjacent to the terminal cisternae of the SR. ${ }^{1}$ The close apposition of the TT and SR membranes brings together the dihydropyridine receptor (DHPR) and the ryanodine receptor (RyR1), two calcium channels residing respectively in each of these two membrane systems. Upon depolarization of the plasma membrane and TTs, the DHPRs, which sense the voltage and are conformationally coupled to RyR1s, induce the RyR1s to open. This opening results in a massive release of $\mathrm{Ca}^{2+}$ from the SR and the sudden raise of intracellular $\mathrm{Ca}^{2+}$ triggers contraction of the muscle fibers. ${ }^{2}$
DHPR controls RyR1 not only during activation (EC coupling) but also at rest, as lack of DHPR results in elevated resting cytoplasmic $\mathrm{Ca}^{2+3}$ and mutations in DHPR result in the malignant hyperthermia syndrome $^{4-6}$ that has been associated with dysregulation of resting cytoplasmic $\mathrm{Ca}^{2+}$ levels. ${ }^{7,8}$

\section{Role of the dihydripyridine receptor in excitation-contraction coupling of skeletal muscle}

EC coupling relies on a supramolecular complex between DHPR, which is the TT voltage sensor, and the RyR1, which is the SR $\mathrm{Ca}^{2+}$ release channel. The distinctive spatial relationship between these two proteins was revealed using freeze fracture shadowing electron microscopy. On the SR terminal cisternae side of the triad junction, large proteins named "feet" have been identified as RyR1. These form ordered 2D arrays, whereby RyR1s contact each other through their corners forming two or three rows along the edge of the terminal cisternae. On the TT side, groups of four particles (tetrads), identified as 


\section{D structure of muscle dihydropyridine receptor}

Eur J Transl Myol - Basic Appl Myol 2015; 25 (1): 27-33

four DHPRs, correlated with the disposition of individual RyR1 particles on the SR. Tetrads overlap alternate RyR1s, leaving an "orphan" RyR1 in between. ${ }^{9}$ The tetrads of DHPR are formed by the specific interaction between each DHPR and each of the four corners of the much larger, prism-shaped homotetrameric RyR1, and do not form in its absence. ${ }^{10}$ Further evidence of the tight interaction between DHPR and RyR1 is the change in intra-tetrad distance when applying an effector known to change the conformation of RyR $1 .^{11}$

The skeletal muscle DHPR is a heteropentamer formed by the $\alpha 1 \mathrm{~s}$ or CaV1.1, $\alpha 2-\delta 1, \beta 1 \mathrm{a}$, and $\gamma$ subunits. $^{12}$ $\mathrm{CaV} 1.1$, an L-type voltage-activated $\mathrm{Ca}^{2+}$ channel, acts as the voltage sensor for EC coupling. ${ }^{13,14}$ Since its $\mathrm{Ca}^{2+}$ channel activity is irrelevant in the EC coupling context, $^{15,16}$ much effort has been dedicated to understand how the DHPR transmits the voltage-sensing signal induced by membrane depolarization to the RyR1. The main candidates that have emerged are the II-III loop of $\alpha 1 \mathrm{~s}$ and the $\beta 1 \mathrm{a}$ subunit: their presence is essential for EC coupling ${ }^{17.19}$ and tetrad formation. ${ }^{20,21}$ Thus, it became important to see how these two entities are organized within the DHPR complex and how is their spatial relationship with respect to the RyR1.

\section{Challenges in the structural determination of the DHPR}

The DHPR has been visualized within the context of the muscle tissue by freeze fracture followed by rotary shadowing as described above. In this technique, the frozen tissue is fractured through the less cohesive membrane planes, exposing the protruding regions of membrane proteins. These protrusions can then be highlighted by heavy metal directional or rotary shadowing and then imaged on the transmission electron microscope (TEM). ${ }^{9}$ The particles however are featureless and the resolution of this technique is limited by the size of the grain of evaporated heavy metal, to around $50 \AA$. Additionally, it is unclear which part of the molecule is represented by the "particle" since the particle size is the same in presence and absence of the $\alpha 2-\delta 1$ subunits even though these constitute about half the molecular weight of the whole complex. $^{22}$

An approach to get higher resolution, at the expense of losing the triad context, has been to extract the DHPR complex from the membrane using detergent, and then to image the solubilized protein using TEM and single-particle 3D reconstruction. Purification and 3D structural determination of membrane proteins has many challenges when compared to soluble proteins, ${ }^{23}$ therefore structures of membrane proteins account for less than $2 \%$ of all protein structures available in the $3 \mathrm{D}$ structure databases. Extraction of the protein from the membrane needs the right amount of detergent: too little detergent lowers the yield of protein, and too much detergent may denature the protein. The choice of detergent is also important; digitonin has been the most widely used for DHPR. After extraction, the DHPR is purified from the other membrane proteins using chromatography that may be further combined with size separation techniques (gel filtration, sucrose gradient). Since DHPR is a heteropentamer, the purification method also has to ensure that all subunits are present at the end of the purification process. Simultaneous heterologous expression of all DHPR subunits yielded functional DHPRs in CHO cells, ${ }^{24}$ however producing sufficient quantity for protein purification could prove difficult. To overcome many of these challenges, one successful approach was to genetically engineer one of the DHPR subunits with a tag for purification, and a second tag for 3D identification. A transgenic mouse expressing this construct was then generated for protein purification. ${ }^{25}$ For 3D structural determination the purified DHPR in solution is then prepared for negative staining or cryoEM, and imaged on the TEM. Negative staining uses heavy metals to provide high contrast of the dehydrated protein at the expense of resolution (up to $\sim 20 \AA$ resolution); cryoEM has less contrast but records images of the protein in its frozen hydrated state and has the potential for higher resolution (up to near atomic resolution). However an additional complication for membrane proteins in the case of cryoEM is the presence of detergent, which reduces image contrast. Single-particle image processing uses the different views of the protein to reconstruct its original shape. This is done by finding the exact orientation in space of each DHPR "particle", back projecting it to obtain its $3 \mathrm{D}$ reconstruction using algorithms related to these used in tomography, and by averaging multiple (thousands) of such particles. Finding the spatial orientation is not trivial when the 3D structure of the protein is not known; specialized algorithms and software tackle this problem. ${ }^{26,27}$ Important factors favoring 3D structural determination are a large size, presence of distinctive features in the protein, and conformational rigidity. While the DHPR has distinctive features and its $\mathrm{MW}$ of $\sim 450 \mathrm{kDa}$ is larger than most proteins, it is slightly small for single particle image processing, and has a flexible region. In this context RyR1, five times larger than DHPR and more rigid, is a much better candidate for this technique, which has enabled its $3 \mathrm{D}$ reconstruction to $10 \AA$ resolution revealing secondary structure. ${ }^{28}$ For DHPR, both negative staining and cryoEM have been applied; the best resolution achieved so far is $19 \AA$ by negative staining. ${ }^{25}$

\section{Three-dimensional structure of the dihydro- pyridine receptor complex}

The 3D structures of skeletal DHPR that have remained consistent with the increase in resolution $^{25,29,30}$ show a main globular body with an 

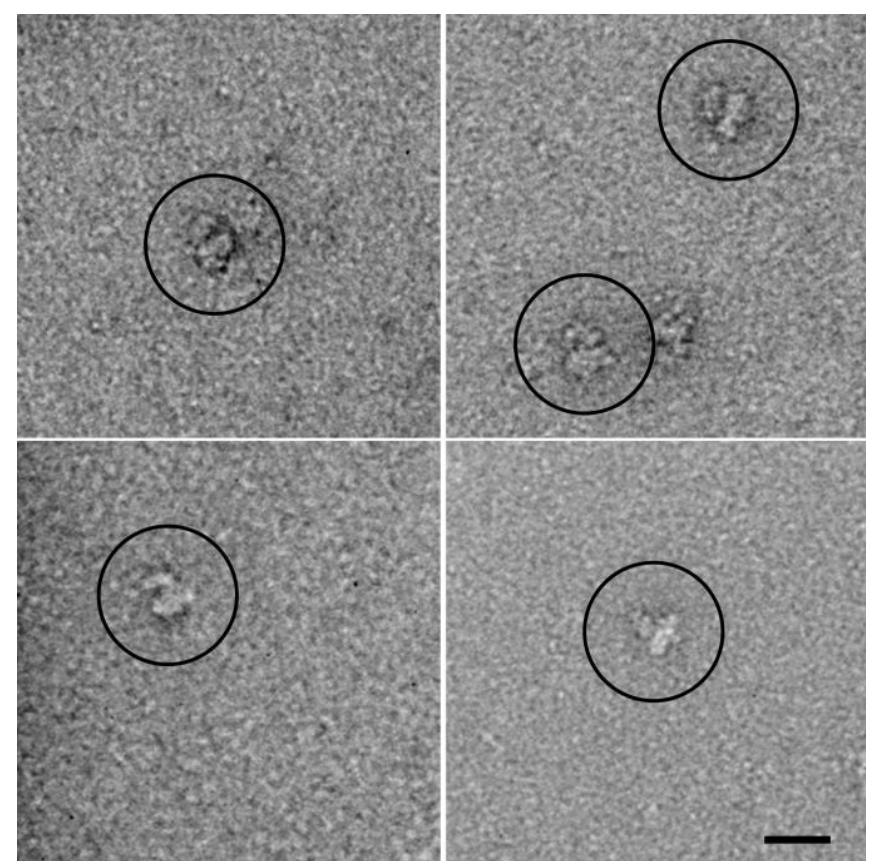

Fig 1. Electron microscopy of negatively stained DHPR. The main globular body with an appendage is visible in the raw images. Scale bar, $20 \mathrm{~nm}$.

appendage emerging from it. The structural description will be centered in our negative staining 3D reconstruction, which has the highest resolution to date (19 $\AA$ ) and enabled placing of the $\alpha 1$ s and $\beta 1$ a subunits for the first time. ${ }^{25}$ The DHPR used to obtain these images had a heterologous $\beta 1$ a subunit that contained an YFP to aid in 3D sub-localization and a biotin-acceptor domain tag that was used for purification; the engineered $\beta 1$ a subunit was expressed in transgenic mouse as indicated above. After purification from the muscle tissue, the DHPR sample was negatively stained, imaged by TEM (Fig. 1), and its $3 \mathrm{D}$ reconstruction was determined by single particle analysis, using around 12,000 particles. Its dimensions are $17 \times 11 \times 8 \mathrm{~nm}^{3}$ for the main body and an appendage that extends $7 \mathrm{~nm}$ from the point of attachment to the main body (Fig. 2A-B). The main body shows a flat oblong shape with five pronounced corners around its contour, and a small protrusion on its base, opposite to the side where the appendage is attached. The engineered YFP, which is directly recognizable in the structure, together with antibody labeling in this and previous works, identifies several subunits and functional regions within the complex. CaV1.1 or $\alpha 1 \mathrm{~s}$ is the largest subunit (176 kDa) and contributes the voltage-sensing feature. It has a similar molecular organization to that found in voltage sensitive $\mathrm{K}^{+}$and $\mathrm{Na}^{+}$channels and it is assumed that the tertiary structure is also the same $;^{31}$ their canonical organization consists of four repeats with six transmembrane each, S1-S6, where S1-S4 form the

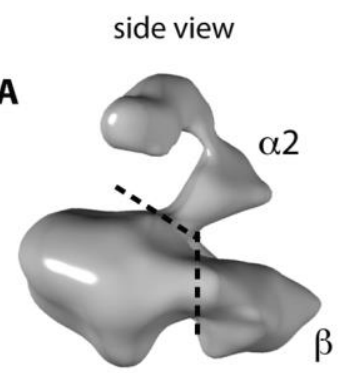

$\alpha 1$ s or Cav1.1

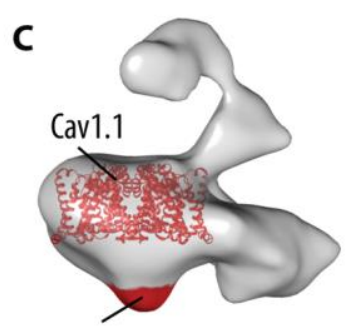

II-III loop
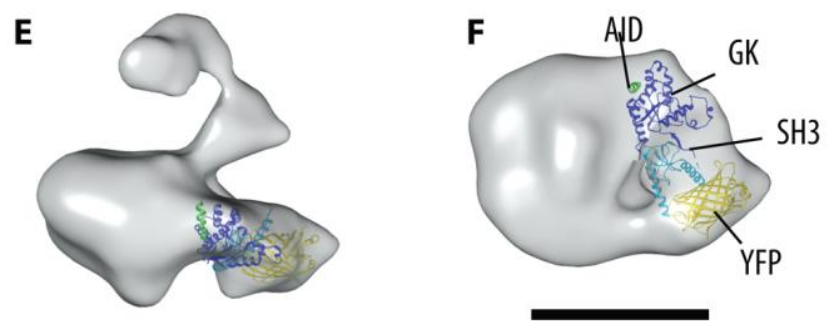

Fig 2. $3 D$ reconstruction of the DHPR and docking of the $\alpha 1 s$ and $\beta$ subunits. A-B. The boundaries of the $\alpha 1 s$, $\alpha 2$ and $\beta$ subunits within the $3 D$ reconstruction of the DHPR are indicated in the two orthogonal views. $C$-D. Molecular boundaries of $\alpha 1$ s: docking of the atomic structure of a representative voltage-gated cation channel $\left(N a v A b^{32}\right)$, and location of the II-III loop. E-F. Docking of the crystal structure of a $\beta$ subunit ${ }^{35}$ with the SH3 and GK domains indicated in light and dark blue, respectively. The barrel-shape of YFP (yellow), engineered in the $N$ terminus of $\beta 1$, is recognizable in the $3 D$ structure. The AID sequence of $\alpha 1 s^{\prime}$ I-II loop (green) that co-crystallized with the $\beta$ subunits makes contact with the $\alpha 1 s$ subunit. According to functional studies, the $\gamma$ subunit should locate at the interphase between $\alpha 1 \mathrm{~s}$ and $\beta$. Scale bar, $10 \mathrm{~nm}$.

peripheral voltage sensors, and S5-S6 from each subunit combine in the center to form the cation channel, forming an overall square prism structure with protruding corners, and a footprint of $9 \times 9 \mathrm{~nm}^{2}$. For each subunit, an additional pore helix between S5-S6 


\section{D structure of muscle dihydropyridine receptor}

Eur J Transl Myol - Basic Appl Myol 2015; 25 (1): 27-33

and an S4-S5 linker do not cross the membrane. We placed the atomic structure of a voltage gated cation channel $^{32}$ in the main body of the TEM derived structure, matching two contiguous square corners separated by $11 \mathrm{~nm}$ (Fig. 2C-D). We attribute the slightly wider dimensions of the TEM structure to the presence of detergent surrounding the transmembrane domain, and to the lower resolution of the TEM structure. Two voltage sensors would be totally exposed to the periphery of the structure while the other two are partially exposed to the periphery and partially interacting with the rest of the main body.

A characteristic of mammalian $\mathrm{CaV}$ channels is that the four repeated units (I-IV) are encoded in a single polypeptide chain of almost 1,900 residues. ${ }^{33}$ This allows for differences in sequence among the four repeats, and importantly, cytoplasmic loops connecting these repeats have distinct structural and functional roles. The I-II loop (residues 334-432) has the alpha-interacting domain (AID) region (residues 357-374) that anchors the $\beta 1$ a subunit. ${ }^{34,35}$ The II-III loop (residues 671-790), longest and partially unfolded, ${ }^{36}$ is an important determinant of EC coupling; ${ }^{17}$ the sequence formed by residues $720-760$ is the critical sequence for EC coupling. ${ }^{18,37}$ The III-IV loop (residues 1066-1117) influences CaV1.1 current but does not appear to be involved in EC coupling interactions with the RyR $1,{ }^{38}$ however its importance is underscored by the fact that mutation at residue 1086 results in malignant hyperthermia. ${ }^{5} \mathrm{CaV} 1.1$ has an extended $C^{\prime}$ domain of $\sim 500$ residues; its distal part is proteolytically cleaved in vivo and in general remains associated through non-covalent interactions. ${ }^{39}$

TEM images of the DHPR incubated with antibodies against the II-III loop showed that these bound to the protuberance emerging from the main body in the center of the region attributed to the transmembrane region of $\mathrm{CaV} 1.1$, and on the opposite side of the appendage, ${ }^{25}$ indicating that this protuberance contains the II-III loop (Fig. 2C-D). This region marks the intracellular side of $\mathrm{CaV} 1.1$ and hence it is plausible that it also contains at least part of the neighboring II-II and III-IV loops, and the $\mathrm{C}^{\prime}$ domain $^{17}$.

$\boldsymbol{\alpha 2}$ and $\boldsymbol{\delta} 1$ subunits The extracellular $\alpha 2$ subunit (147 $\mathrm{kDa}$ ) was previously assigned to the appendage emerging from the main body, using antibody labeling. ${ }^{29,30}$ Together with the identification of the II-III loop within the CaV1.1 subunit, this establishes the orientation of the DHPR 3D structure within the triad junction. Compared with the main body of the DHPR, the size and shape of the appendage attributed to $\alpha 2$ is very threshold-dependent. This is indicative of variability within this region, which is compatible with its heavy glycosylation, and may also be an indicator of flexibility.

The $\alpha 2$ and $\delta 1$ subunits are encoded by a single gene; ${ }^{40}$ the product is later cleaved but remains disulfide-bonded. Thus the single-pass transmembranal $\delta 1$ subunit $(24 \mathrm{kDa})$ must be at the base of the $\alpha 2$ subunit, anchoring it to the membrane.

$\beta 1 \mathrm{a}$ subunit The position of the intracellular $\beta 1 \mathrm{a}$ subunit $(56 \mathrm{kDa})$ was established by identification of the typical barrel shape corresponding to YFP (Fig. $2 \mathrm{~F}$ ), engineered at $\beta 1$ a's $\mathrm{N}$ terminus. ${ }^{25}$ This localization is compatible with previous antibody labeling. ${ }^{29,30}$ The atomic structure of the core region of the $\beta 2 \mathrm{a}$ isoform, encompassing the Src Homology 3 (SH3) domain and a guanylate kinase (GK) domain, ${ }^{35}$ with $84 \%$ identity with $\beta 1$ a, was docked into the $3 \mathrm{D}$ reconstruction of the DHPR complex obtained by TEM taking several factors into account. The alpha-binding pocket of the $\beta$ subunit, which interacts with the AID binding region within the I-II loop of $\alpha 1 \mathrm{~s}$, was situated adjacent to $\alpha 1 \mathrm{~s}$ in a position appropriate for this inter-subunit interaction to occur. The $\mathrm{SH} 3$ in the $\mathrm{N}^{\prime}$ region of the $\beta$ subunit was placed adjacent to the identifiable YFP engineered in the N' region. Finally, the two prominent lobes of the GK domain were fit with the two outward spikes in the 3D volume of DHPR ${ }^{25}$ (Fig. 2E-F). In addition that the choice of location and orientation satisfy all the above conditions, further confidence in this model comes from the fact that the docking is close fitting when seen from all directions (see Supplemental movie)

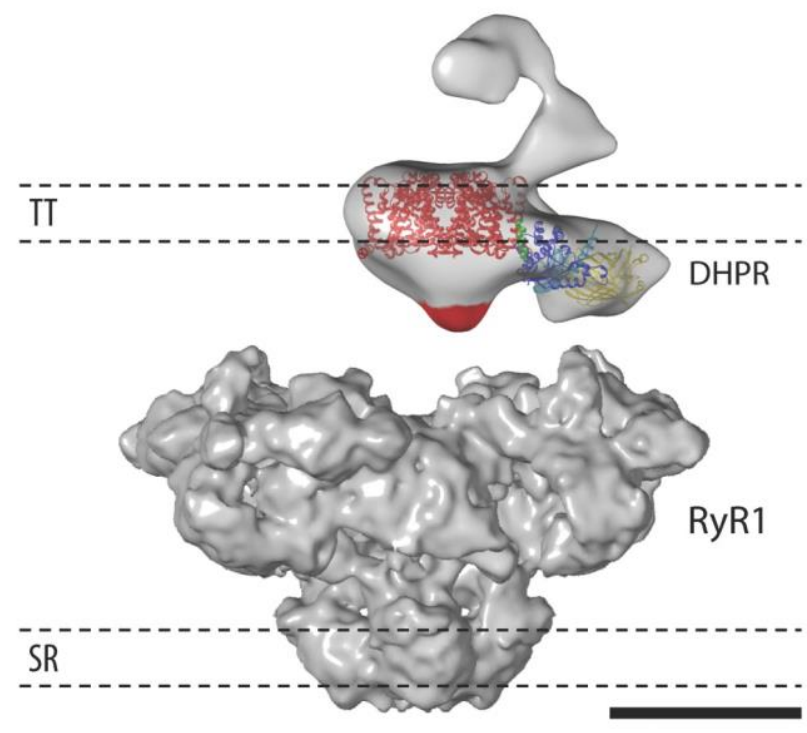

Fig 3. Approximate quaternary arrangement of the DHPR and the RyRl in the triad junction. In this disposition, the II-III loop and the $\beta$ subunit of the DHPR, domains important for EC coupling, are proximal to the RyRl. In addition, $\beta 1 a$ is located just under the membrane and besides $\alpha 1 s$, in agreement with its role of trafficking $\alpha 1$ s to the membrane in the vicinity of RyRl. The TT and SR membrane boundaries are indicated. The two proteins are represented at the same scale. Scale bar, $10 \mathrm{~nm}$. 


\section{D structure of muscle dihydropyridine receptor}

Eur J Transl Myol - Basic Appl Myol 2015; 25 (1): 27-33

$\gamma$ subunit The $\gamma$ subunit $(34 \mathrm{kDa})$, with four transmembrane domains, must be in one of the empty spaces near $\alpha 1 \mathrm{~s}$, as it is known to interact with this subunit. $^{41}$

In the context of the quaternary interactions with the RyR1, the DHPR would be facing the RyR1 across the junctional triadic cleft. On the SR side, the RyR1's cytoplasmic domain (i.e. the foot structure) occupies $120 \AA$ of the cleft, practically bridging the space between the two membranes. On the TT side, the II-III loop of CaV1.1 is sandwiched between the rest of the DHPR and the RyR1, in full agreement with its vital role in EC coupling. ${ }^{17}$ The $\beta 1$ a subunit location, adjacent to CaV1.1 and sandwiched between the TT membrane and the RyR1, with potentially large areas of interface, is compatible with its functions of trafficking CaV1.1 to the membrane ${ }^{42}$ and control of EC coupling, ${ }^{19}$ possibly indirectly by determining the DHPR-RyR 1 association. ${ }^{21}$ Figure 3 summarizes how the RyR1, the DHPR and its more important EC coupling domains would be located relative to each other in the context of the triad junction.

It is expected that higher resolution structures, combined with functional studies and high electron tomography, will, in the future, provide a picture of the quaternary assembly of DHPR and RyR1 at the atomic level, and enable a more thorough understanding of the EC coupling mechanism at the molecular level. Recent advancements in membrane protein stabilization (i.e. using lipid mimics or nanodisks) and in TEM (most notably the installation of direct electron detectors) should help in the single-particle 3D structural determination of the DHPR and RyR1 at much higher resolution. $^{43}$

\section{Note added in proof}

While this paper was in press, the near-atomic structure of RyRl was solved at $3.8 \AA$ by cryoEM. ${ }^{44}$

\section{Acknowledgement}

This work was supported by American Heart Association grant 14GRNT19660003.

\section{Corresponding Author}

Montserrat Samsó. Department of Physiology and Biophysics, 1101 E. Marshall St., Room 3-009, Richmond, VA 23298, U.S.A.

Telephone: (804) 828-8728; Fax: (804) 828-9492

E-mail address: msamso@vcu.edu

\section{References}

1. Flucher BE. Structural analysis of muscle development: transverse tubules, sarcoplasmic reticulum and the triad. Dev Biol 1992;154:245-60.

2. Schneider MF. Membrane charge movement and depolarization-contraction coupling. Annu Rev Physiol 1981;43:507-7.
3. Eltit JM, Li H, Ward CW, et al. Orthograde dihydropyridine receptor signal regulates ryanodine receptor passive leak. Proc Natl Acad Sci U S A 2011;108:7046-51.

4. Pirone A, Schredelseker J, Tuluc P, et al. Identification and functional characterization of malignant hyperthermia mutation T1354S in the outer pore of the Cavalpha1S-subunit. Am J Physiol Cell Physiol 2010;299:C1345-54.

5. Weiss RG, O'Connell KM, Flucher BE, et al. Functional analysis of the $\mathrm{R} 1086 \mathrm{H}$ malignant hyperthermia mutation in the DHPR reveals an unexpected influence of the III-IV loop on skeletal muscle EC coupling. Am J Physiol Cell Physiol 2004;287:C1094-102.

6. Eltit JM, Bannister RA, Moua O, et al. Malignant hyperthermia susceptibility arising from altered resting coupling between the skeletal muscle L-type $\mathrm{Ca}^{2+}$ channel and the type 1 ryanodine receptor. Proc Natl Acad Sci U S A 2012;109:7923-8.

7. Tong J, McCarthy TV, MacLennan DH. Measurement of resting cytosolic $\mathrm{Ca}^{2+}$ concentrations and $\mathrm{Ca}^{2+}$ store size in HEK-293 cells transfected with malignant hyperthermia or central core disease mutant $\mathrm{Ca}^{2+}$ release channels. J Biol Chem 1999;274: 693-702.

8. Yang T, Esteve E, Pessah IN, et al. Elevated resting $[\mathrm{Ca}(2+)](\mathrm{i})$ in myotubes expressing malignant hyperthermia RyR1 cDNAs is partially restored by modulation of passive calcium leak from the SR. Am J Physiol Cell Physiol 2007;292:C1591-8.

9. Block BA, Imagawa $\mathrm{T}$, Campbell KP, Franzini-Armstrong C. Structural evidence for direct interaction between the molecular components of the transverse tubule/sarcoplasmic reticulum junction in skeletal muscle. J Cell Biol 1988;107:2587-600.

10. Protasi F, Franzini-Armstrong C, Allen PD. Role of ryanodine receptors in the assembly of calcium release units in skeletal muscle. J Cell Biol 1998; 140:831-42.

11. Paolini C, Fessenden JD, Pessah IN, et al. Evidence for conformational coupling between two calcium channels. Proc Natl Acad Sci U S A 2004;101:12748-52.

12. Leung AT, Imagawa $\mathrm{T}$, Block $\mathrm{B}$, et al. Biochemical and ultrastructural characterization of the 1,4-dihydropyridine receptor from rabbit skeletal muscle. Evidence for a 52,000 Da subunit. J Biol Chem 1988;263:994-1001.

13. Rios E, Brum G. Involvement of dihydropyridine receptors in excitation-contraction coupling in skeletal muscle. Nature 1987;325:717-20.

14. Tanabe T, Beam KG Powell JA, Numa S. Restoration of excitation-contraction coupling 


\section{D structure of muscle dihydropyridine receptor}

Eur J Transl Myol - Basic Appl Myol 2015; 25 (1): 27-33

and slow calcium current in dysgenic muscle by dihydropyridine receptor complementary DNA. Nature 1988;336:134-9.

15. Garcia J, Tanabe T, Beam KG. Relationship of calcium transients to calcium currents and charge movements in myotubes expressing skeletal and cardiac dihydropyridine receptors. J Gen Physiol 1994;103:125-47.

16. Armstrong CM, Bezanilla FM, Horowicz P.Twitches in the presence of ethylene glycol bis( -aminoethyl ether)-N,N'-tetracetic acid. Biochim Biophys Acta 1972;267:605-8.

17. Tanabe T, Beam KG, Adams BA, et al. Regions of the skeletal muscle dihydropyridine receptor critical for excitation-contraction coupling. Nature 1990;346:567-9.

18. Kugler G, Weiss RG, Flucher BE, Grabner, M. Structural requirements of the dihydropyridine receptor alpha1S II-III loop for skeletal-type excitation-contraction coupling. J Biol Chem 2004;279:4721-8.

19. Coronado R, Ahern CA, Sheridan DC, et al. Functional equivalence of dihydropyridine receptor alpha1S and beta1a subunits in triggering excitation-contraction coupling in skeletal muscle. Biol Res 2004;37:565-75.

20. Takekura H, Paolini C, Franzini-Armstrong C, et al. Differential contribution of skeletal and cardiac II-III loop sequences to the assembly of dihydropyridine-receptor arrays in skeletal muscle. Mol Biol Cell 2004;15:5408-19.

21. Schredelseker J, Di Biase V, Obermair GJ, et al. The beta 1a subunit is essential for the assembly of dihydropyridine-receptor arrays in skeletal muscle. Proc Natl Acad Sci U S A 2005;102:17219-24.

22. Gach MP, Cherednichenko G, Haarmann C, et al. Alpha2 delta1 dihydropyridine receptor subunit is a critical element for excitation-coupled calcium entry but not for formation of tetrads in skeletal myotubes. Biophys J 2008;94:3023-34.

23. Torres J, Stevens TJ, Samso M. Membrane proteins: the 'Wild West' of structural biology. Trends Biochem Sci 2003;28:137-44.

24. Takekura H, Takeshima H, Nishimura S, et al. Co-expression in CHO cells of two muscle proteins involved in excitation-contraction coupling. J Muscle Res Cell Motil 1995;16:465-80.

25. Szpyt J, Lorenzon N, Perez CF, et al. Three-Dimensional Localization of the alpha and beta Subunits and of the II-III Loop in the Skeletal Muscle L-type $\mathrm{Ca}^{2+}$ Channel. J Biol Chem 2012;287:43853-61.

26. Shaikh TR, Gao H, Baxter WT, et al. SPIDER image processing for single-particle reconstruction of biological macromolecules from electron micrographs. Nat Protoc 2008;3:1941-74.

27. Ludtke SJ, Baldwin PR, Chiu W. EMAN: semiautomated software for high-resolution single-particle reconstructions. J Struct Biol 1999;128:82-97.

28. Samso M, Wagenknecht $\mathrm{T}$, Allen PD. Internal structure and visualization of transmembrane domains of the RyR1 calcium release channel by cryo-EM. Nat Struct Mol Biol 2005;12:539-44.

29. Murata K, Nishimura S, Kuniyasu A, Nakayama H. Three-dimensional structure of the alpha1-beta complex in the skeletal muscle dihydropyridine receptor by single-particle electron microscopy. J Electron Microsc (Tokyo) 2010;59:215-26.

30. Wolf M, Eberhart A, Glossmann H, et al. Visualization of the domain structure of an L-type $\mathrm{Ca}^{2+}$ channel using electron cryo-microscopy. J Mol Biol 2003;332:171-82.

31. Yu FH, Yarov-Yarovoy V, Gutman GA, Catterall WA. Overview of molecular relationships in the voltage-gated ion channel superfamily. Pharmacol Rev 2005;57:387-95.

32. Payandeh J, Scheuer T, Zheng N, Catterall, WA. The crystal structure of a voltage-gated sodium channel. Nature 2011;475:353-8.

33. Catterall WA, Perez-Reyes E, Snutch, TP Striessnig. J International Union of Pharmacology. XLVIII. Nomenclature and structure-function relationships of voltage-gated calcium channels. Pharmacol Rev 2005;57:411-25.

34. Pragnell M, De Waard M, Mori Y, et al. Calcium channel beta-subunit binds to a conserved motif in the I-II cytoplasmic linker of the alpha 1-subunit. Nature 1994;368:67-70.

35. Opatowsky Y, Chen CC, Campbell K, PHirsch, JA. Structural analysis of the voltage-dependent calcium channel beta subunit functional core and its complex with the alpha 1 interaction domain. Neuron 2004;42:387-99.

36. Casarotto MG, Cui Y, Karunasekara Y, et al. Structural and functional characterization of interactions between the dihydropyridine receptor II-III loop and the ryanodine receptor. Clin Exp Pharmacol Physiol 2006;33:1114-7.

37. Nakai J, Tanabe T, Konno T, et al. Localization in the II-III loop of the dihydropyridine receptor of a sequence critical for excitation-contraction coupling. J Biol Chem 1998;273:24983-6.

38. Bannister RA, Grabner M,Beam KG. The alpha(1S) III-IV loop influences 1,4-dihydropyridine receptor gating but is not directly involved in excitation-coupling interactions with the type 1 ryanodine receptor. $\mathbf{J}$ Biol Chem 2008;283:23217-23. 


\section{D structure of muscle dihydropyridine receptor}

Eur J Transl Myol - Basic Appl Myol 2015; 25 (1): 27-33

39. Hulme JT, Konoki K, Lin TW, et al. Sites of proteolytic processing and noncovalent association of the distal C-terminal domain of CaV1.1 channels in skeletal muscle. Proc Natl Acad Sci U S A 2005;102:5274-9.

40. De Jongh KS, Warner C, Catterall WA. Subunits of purified calcium channels. Alpha 2 and delta are encoded by the same gene. J Biol Chem 1990;265:14738-41.

41. Arikkath J, Chen CC, Ahern C, Allamand, V et al. Gamma 1 subunit interactions the skeletal muscle L-type voltage-gated calcium channels. J Biol Chem 2003;278:1212-9.
42. Gregg RG, Messing A, Strube C, et al. Absence of the beta subunit (cchb1) of the skeletal muscle dihydropyridine receptor alters expression of the alpha 1 subunit and eliminates excitationcontraction coupling. Proc Natl Acad Sci U S A 1996;93:13961-6.

43. Cabra V, Samso M. Do's and Don'ts of Cryo-electron microscopy: A primer on sample preparation and high quality data collection for macromolecular 3D reconstruction. J Vis Exp 2015;95: e52311.

44. Yan Z, Bai X-C, Yan C, et al. Structure of the rabbit ryanodine receptor RyR1 at near-atomic resolution. Nature 2014;517:50-5. 